\title{
Rasgos morfológicos regenerativos en una comunidad de especies leñosas en un bosque seco tropical tumbesino
}

\author{
José Miguel Romero-Saritama ${ }^{1 *} \&$ César Pérez-Rúiz ${ }^{2}$ \\ 1. Departamento de Ciencias Naturales. Universidad Técnica Particular de Loja, San Cayetano Alto, Marcelino \\ Champagnat, 1101608, Loja, Ecuador; jmromero@utpl.edu.ec \\ 2. Departamento de Biotecnología y Biología Vegetal. Escuela Técnica Superior de Ingenieros Agronomos Universidad \\ Politécnica de Madrid, España; cesar.perez@upm.es \\ * Correspondencia
}

Recibido 27-VII-2015. Corregido 14-I-2016. $\quad$ Aceptado 16-II-2016.

\begin{abstract}
Regenerative morphological traits in a woody species community in Tumbesian tropical dry forest. The study of functional morphological traits enables us to know fundamental aspects of the dynamics of plant communities in local and global habitats. Regenerative morphological traits play an important role in defining plant history and ecological behavior. Seed and fruit characteristics determine to a large extent the patterns for dispersal, germination, establishment and seedling recruitment a given species exhibits on its natural habitat. Despite their prominent role, seed and fruit traits have been poorly studied at the community level of woody plant species in neo-tropical dry forests. In the present study we aimed at i) evaluate the functional role of morphological traits of seeds, fruits and embryo in woody plant species; ii) determine which are the morphological patterns present in seeds collected from the community of woody species that occur in neo-tropical dry forests; and iii) compare woody plant species seed mass values comparatively between neo-tropical dry and tropical forests. To do so, mature seeds were collected from 79 plant species that occur in the Tumbesian forest of Southwest Ecuador. The studied species included the 42 and 37 most representative tree and shrubbery species of the Tumbesian forest respectively. A total of 18 morphological traits (seven quantitative and 11 qualitative) were measured and evaluated in the seeds, fruits and embryos of the selected species, and we compared the seeds mass with other forest types. Our results showed a huge heterogeneity among traits values in the studied species. Seed mass, volume and number were the traits that vary the most at the community level, i.e. seed length ranged from 1.3 to $39 \mathrm{~mm}$, and seed width from 0.6 to $25 \mathrm{~mm}$. Only six embryo types were found among the 79 plant species. In $40 \%$ of the cases, fully developed inverted embryos with large and thick cotyledons to store considerable amount of nutrients were recorded. We concluded that highly variable and functionally complementary morphological traits occur among the studied woody plants of the Tumbesian dry forest. The latter favors a plethora of behavioral mechanisms to coexist among woody species of the dry forest in response to the environmental stress that is typical of arid areas. Rev. Biol. Trop. 64 (2): 859-873. Epub 2016 June 01.
\end{abstract}

Key words: Ecuador, tropical dry forest, trees, shrubs, plant embryos, fruist.

El estudio de rasgos funcionales en las plantas se vuelve cada día más importante en la ecología de las comunidades (Kröber, Böhnke, Welk, Wirth, \& Bruelheide, 2012). Estos pueden predecir el resultado de ensamble de la comunidad (Shipley, Vile, \& Garnier, 2006; Laughlin, Joshi, Van, Bastow, \& Fulé, 2012), la dinámica de la vegetación mundial (Scholze, Knorr, Arnell, \& Prentice, 2006) y el ritmo de los procesos de las especies dentro de los ecosistemas (Garnier et al., 2004). Sin embargo, todavía muchas especies vegetales faltan por describirse taxonómicamente a nivel mundial, especialmente en hábitat biodiversos como son las zonas tropicales. Trabajos recientes sugieren que nuestra comprensión de las comunidades ecológicas puede progresar más rápidamente a través de un enfoque en los rasgos funcionales 
de los organismos (Bihn, Gebauer, \& Brandl, 2010; Yates, Andrew, Binns, \& Gibb, 2014).

Las comunidades vegetales de los bosques tropicales poseen una gran diversidad de rasgos morfológicos que forman parte de la historia de vida de las plantas (Andrew, Hart, Jung, Hemmings, \& Terblanche, 2013). La variación encontrada en cada uno de los componentes de las especies incluyendo frutos y semillas, posiblemente sea una respuesta al ambiente donde evolucionaron (Westoby, Falster, Moles, Vesk, \& Wright, 2002); sin embargo, todavía no se han estudiado todos los hábitats. El estudio de rasgos regenerativos (frutos y semillas) son un elemento importante y crítico en la ecología, evolución e historia de las comunidades vegetales (Vandelook, Verdu, \& Honnay, 2012a), debido a que afectan directamente a procesos de adaptación, dispersión, germinación, colonización y establecimiento de las plántulas (Vandelook, Janssens, \& Probert, 2012b) en los diferentes ecosistemas. Formando parte de los ecosistemas tropicales, se encuentran los bosques secos, que comprenden el $42 \%$ de los bosques tropicales (Chaturvedi, Raghubanshi, \& Singh, 2011); estos constituyen comunidades de árboles que crecen en climas cálidos a calientes con una marcada estacionalidad en lluvia, y entre dos y seis meses de sequía cada año, durante el cual la proporción de evaporación potencial para lluvia es mayor que uno (Pennington, Lewis, \& Ratter, 2006). La estacionalidad pronunciada en los bosques secos controla la estructura de la vegetación (Gritti et al., 2010), afectando los patrones de producción de semillas, germinación, supervivencia y desarrollo de las plántulas, por lo tanto, el período de crecimiento favorable en zonas áridas suele estar restringida a la corta temporada de lluvias cuando se espera que las semillas germinen y se establezcan las plántulas (Donohue, Rubio de Casas, Burghardt, Kovach, \& Willis, 2010; Metz, Liancourt, Kigel, Sternberg, \& Tielbörger, 2010).

Lamentablemente, los bosques secos tropicales a pesar de su importancia se han reducido a menos del $0.1 \%$ de su extensión original, y en América del Sur se encuentran es un estado crítico, están más amenazados, menos estudiados y protegidos (Portillo-Quintero \& SánchezAzofeifa, 2010) que los bosques húmedos y muy húmedos (Aide et al., 2012). Además, muchos de los bosques secos se han convertido en pastizales, bosques secundarios abiertos, sabanas, han sido quemados y convertidos en ranchos de ganado o tierras agrícolas (Aide et al., 2012; Blackie et al,. 2014). Actualmente, podemos encontrar bosques secos en un mosaico de parches cercanos con dosel abierto, relativamente inalterados y perturbados en varias escalas espaciales que constituyen una marcada heterogeneidad en términos de irradiación, temperatura, humedad y velocidad de liberación de nutrientes a partir de la descomposición de la hojarasca (Quesada \& Stoner, 2004).

En Ecuador, los bosques secos están restringidos a lo largo de la costa desde el norte hasta el sur de la provincia de Loja y hacia los valles secos interandinos (Aguirre, LinaresPalomino, \& Kvist, 2006). Los bosques secos de la costa forman parte de la región tumbesina compartida entre Ecuador y Perú con una extensión de $135000 \mathrm{~km}^{2}$, caracterizados por poseer una alta diversidad y gran cantidad de especies endémicas (Aguirre \& Kvist, 2005; LinaresPalomino, Oliveira-Filho \& Pennington, 2011). Al sur occidente del Ecuador se encuentra la mayor superficie de bosques secos del país que han sido relativamente bien conservados y que no sufrieron grandes procesos de deforestación (Espinosa, Cabrera, Luzuriaga, \& Escudero, 2011). El área es conocida por su alto nivel de endemismo de especies de flora (Aguirre et al., 2006) e incluye tierras bajas, estribaciones occidentales bajas de la cordillera de los andes y los valles interandinos del sur (Aguirre \& Kvist, 2005). Sin embargo, actualmente los bosques secos tumbesinos presentes en el sur del Ecuador también han sido expuestos a grandes presiones antropogénicas, y es evidente observar la transformación de estos ecosistemas en zonas de pastizales y áreas destinadas para la agricultura, y el turismo generado con el florecimiento de los guayacanes (Tabebuia chrysantha), siendo fundamental realizar investigación y conservación de las especies en 
este ecosistema frágil. Si bien es cierto que en los últimos años se han realizado algunos estudios florísticos en la región tumbesina donde se destacan los trabajos realizados por LinaresPalomino, Aguirre-Mendoza y Gonzales-Inca (2010), Espinosa et al. (2011), Jara-Guerrero, De la Cruz y Méndez (2011) y Espinosa, De la Cruz, Luzuriaga y Escudero (2012), para mejorar el conocimiento de la estructura y funcionamiento de los bosques secos, así como los patrones de dispersión de semillas, todavía es necesario e importante identificar y conocer los diferentes rasgos morfológicos de semillas presentes en los bosques secos tumbesinos.

Partiendo de la premisa que los rasgos regenerativos cumplen un rol fundamental en la ecología de las especies y que han sido poco o casi nada estudiados en zonas áridas (Metz et al., 2010), más aún, en los bosques secos tumbesinos, los objetivos de nuestro estudio fueron: a) explorar aspectos de la ecología de semillas a nivel de una comunidad de especies leñosas mediante la descripción y evaluación de rasgos morfológicos funcionales en frutos, semillas y embriones, b) determinar qué patrones morfológicos caracterizan a las semillas de especies leñosas distribuidas en uno de los remanentes de bosque seco más importantes de la región tumbesina, c) analizar la masa de la semillas con respecto a otros bosques tropicales. Los resultados generados en la presente investigación son una base fundamental para contribuir al conocimiento de la dinámica de los bosques secos tumbesinos, y a la generación de pautas para la conservación de sus especies, basado en rasgos morfológicos regenerativos.

\section{MATERIALES Y MÉTODOS}

Área de estudio: El estudio se realizó en la provincia de Loja al Sur Occidente del Ecuador (4¹9'39" - 4'01'40" S y 80¹9'00" - 7941'40" W) a una altitud de 250 a 1200 m s.n.m. La zona comprende remanentes de bosques secos tropicales deciduos y semideciduos con mayor superficie en el Ecuador y con vegetación poco alterada (Espinosa et al., 2011). El clima del lugar es bien marcado, se caracteriza por una estación lluviosa desde diciembre a abril, y una estación seca de mayo a noviembre, precipitación media anual de 500 $\mathrm{mm}$ con una temperatura media anual de 20 a $26{ }^{\circ} \mathrm{C}$. (Espinosa et al., 2011).

La vegetación en la zona se caracteriza por una alta diversidad de plantas vasculares endémicas, con árboles y arbustos dispersos en todo el lugar, algunos pierden sus hojas exponiendo solamente sus frutos en época seca como por ejemplo: Ceiba trichistandra (A. Gray) Bakh., Eriotheca ruizii (K. Schum.) A. Robyns, (Bombacaceae), Cochlospermum vitifolium Spreng. (Cochlospermaceae). En cambio, especies como Juglans neotropica Diels (Juglandaceae) y Siparuna eggersii Hieron. (Monimiaceae) se encuentran en peligro, Cavanillesia platanifolia Kunth se encuentra casi amenazada y Cedrela odorata L., Swietenia macrophylla King (Meliaceae) en estado vulnerable (IUCN, 2015).

Recolección de semillas: Del 2010 al 2014 se recolectaron frutos con semillas maduras (durante el proceso de dispersión natural) de entre ocho a diez individuos de 79 especies leñosas (ver Anexo 1) correspondientes a 31 familias representativas de los bosques secos, 42 especies de árboles y 37 correspondieron a arbustos. La recolecta se restringió a aquellas especies más abundantes y de interés especial por su uso. La nomenclatura para la identificación familias, especies y nombres científicos fue en base a The International Plant Names Index (disponible en http://www.ipni.org/). Los frutos y semillas recolectadas de todas las especies fueron llevados al laboratorio del Banco de Germoplasma de la Universidad Técnica Particular de Loja (UTPL) para su procesamiento y análisis.

Rasgos de semillas: Utilizamos un total de 18 rasgos morfológicos regenerativos entre cuantitativos y cualitativos, tres en frutos, 13 en semillas, y dos características generales de las especies (Cuadro 1) en una muestra de 50 frutos y semillas por especie. Los frutos fueron clasificados en dos categorías según 
CUADRO 1

Tipo y escala de rasgos morfológicos regenerativos en especies de bosque seco

TABLE 1

Type and scale of regenerative morphological traits in dry forest species

\begin{tabular}{lcc}
\multicolumn{1}{c}{ Rasgo } & Escala & $\begin{array}{c}\text { Número de } \\
\text { especies }\end{array}$ \\
Largo $(\mathrm{mm})$ & continua & 79 \\
Ancho $(\mathrm{mm})$ & continua & 79 \\
Volumen $\left(\mathrm{mm}^{3}\right)$ & continua & 79 \\
Masa $(\mathrm{g})$ & continua & 79 \\
Largo embrión $(\mathrm{mm})$ & continua & 75 \\
Apéndice & dicotómica & 79 \\
Forma & nominal & 79 \\
Areola & dicotómica & 79 \\
Tipo testa & dicotómica & 79 \\
Textura testa & dicotómica & 79 \\
Tipo embrión & nominal & 78 \\
Función embrión & dicotómica & 77 \\
Endopermo & dicotómica & 77 \\
Hábito & dicotómica & 79 \\
Tamaño especie (m) & continua & 79 \\
Tipo fruto & dicotómica & 79 \\
Tipo dispersión & nominal & 79 \\
Número semillas/fruto & discreta & 71 \\
\hline
\end{tabular}

su consistencia: secos y carnosos. Durante la extracción de semillas determinamos el número de semillas por fruto. Para el tipo de dispersión nos basamos en Jara-Guerrero et al. (2011) y se clasificó en tres tipos generales: zoocoria, autocoria y anemocoria.

Para determinar el tamaño de las semillas se midió el largo, ancho y grosor en mm usando un escalímetro digital de dos dígitos. En semillas demasiado pequeñas así como para los embriones, se tomaron fotografías y se procedió a medir mediante el software imagen $\mathrm{J}$ (disponible en http://imagej.nih.gov/ij/), usado además para medir el área foliar (Newton et al., 2013). Además, calculamos el volumen y la masa de cada semilla para todas las especies. La testa de las semillas se clasificó como lisa o rugosa en función de la superficie de la testa. La textura de la testa fue catalogada como dura o blanda de acuerdo a si esta se rompía o no manualmente. Para identificar rasgos internos se realizaron cortes transversales y longitudinales a las semillas, y se determinó la presencia o ausencia del endospermo, así como el tipo de embrión en base a Martin (1946), clasificación que hasta la actualidad se sigue utilizando como una referencia en estudios de evolución, morfología y dormancia de semillas (Finch-Savage \& Leubner-Metzger, 2006). La función del embrión se evaluó de acuerdo a los cotiledones siguiendo la metodología de Kitajima (1996), y se determinó solamente en cotiledones de almacenamiento, si estos eran gruesos carnosos, o como foliares si eran finos o pequeños, dando la apariencia a ser órganos fotosintéticos (finas hojas) más que de almacenamiento. El tamaño de la especie representa la altura de la planta en metros, esta medida fue tomada de diferentes referencias bibliográficas de estudios realizados en la zona. La determinación de árbol y arbusto fue de acuerdo a Aguirre et al. (2006).

\section{Comparaciones entre diferentes tipos de} bosque: El promedio de la masa de las semillas de las especies del bosque seco en estudio, la comparamos con información bibliográfica disponible para la masa de semillas de especies presentes en otros tipos de bosques, tres bosques neotropicales y un bosque seco tropical presente en la India (Cuadro 2). La denominación del tipo de bosque y la condición en el momento de la medición de las semillas ya sea seca o fresca se mantuvo de acuerdo a la clasificación original donde se realizó el estudio y se seleccionaron solamente especies que correspondan a árboles o arbustos, en algunos casos donde la medida de las semillas era en miligramos, fueron convertidos a gramos para tener una sola medida.

Se realizó un análisis descriptivo en todos los rasgos medidos y determinamos la distribución de los valores cualitativos mediantes histogramas. Utilizamos la prueba de chi cuadrado $\left[\mathrm{x}^{2}=\sum(\mathrm{fo}-\mathrm{fe})^{2} / \mathrm{fe}\right]$, donde fo $=$ significa frecuencia observada y fe $=$ frecuencia esperada, para comparar atributos dentro de rasgos cualitativos. Para determinar si existen 
CUADRO 2

Tipos de bosques tropicales donde se obtuvo información de la masa de las semillas

TABLE 2

Types of tropical forests where seed mass information was obtained

\begin{tabular}{lccl}
\multicolumn{1}{c}{ Tipo de bosque } & Sitio & Número de especies & \multicolumn{1}{c}{ Referencia } \\
Bosque semideciduo (BSD) & Panamá & 104 & Daws, Garwood y Pritchard (2006) \\
Bosque siempre verde (BSV) & Cuba & 40 & Sánchez, Muñoz y Montejo (2009) \\
Bosque húmedo tropical (BHT) & Perú & 165 & Foster y Janson (1985) \\
Bosque seco (BS) & India & 37 & Khurana, Sagar y Singh (2006) \\
\hline
\end{tabular}

diferencias en la media de la masa de las semillas entre los tipos de bosque usamos la prueba de Kruskal Wallis, y se realizó un análisis de comparación multiple mediante el test de Tukey $(\mathrm{p}<0.05)$ para identificar en qué tipos de bosque se presentó diferencia significativa en la masa de las semillas. Todos los análisis fueron realizados con el software $\mathrm{R}$ ( $\mathrm{R}$ environment Core Team, 2013).

\section{RESULTADOS}

Rasgos morfológicos cuantitativos: Existió gran heterogeneidad en los resultados de rasgos cuantitativos medidos (Fig. 1). Sin embargo, existen patrones morfológicos que caracterizan a las semillas de las 79 especies de bosque seco (Fig. 2). Se encontró semillas muy pequeñas desde $1.3 \mathrm{~mm}$ (ej. Acnistus arborencens Schltdl.) a $39 \mathrm{~mm}$ de largo (ej. Cavanillesia platanifolia), obteniendo como promedio final $10.8 \mathrm{~mm}$ con una alta variación $( \pm 7.7)$. En cambio, el promedio del ancho de las semillas correspondió a $6.18 \mathrm{~mm} \mathrm{( \pm}$ 4.3), con anchos entre 0.6 (ej. Chromolaena odorata (L.) R.M.King \& H.Rob.) a $25 \mathrm{~mm}$ (Hura crepitans L.). El volumen fue uno de los rasgos que presentó alta distribución y variabilidad, determinando valores desde $0.28 \mathrm{~mm}^{3}$ en $A$. arborescens a $5900 \mathrm{~mm}^{3}$ en semillas de Geoffroea spinosa Jacq. La masa de las semillas también presentó gran dispersión en sus resultados variando desde 0.0001 a más de $5 \mathrm{~g}$ por semilla. La gran diversidad de semillas por fruto también fue evidente: el $30 \%$ de las especies tienden a producir una sola semilla
( ej. Bursera graveolens Triana \& Planch., Celtis iguanaea (Jacq.) Sarg., Cordia alliodora (Ruiz \& Pav.) Oken, Ziziphus thyrsiflora Benth. Gallesia integrifolia (Spreng.) Harms, entre otras), el $25 \%$ de las especies produce entre 3 y 10 semillas por fruto, pocas especies como Ochroma pyramidale (Cav. ex Lam.) Urb. alcanzaron hasta 500 semillas por fruto. El promedio del largo del embrión fue $8.10 \pm$ $6.3 \mathrm{~mm}$. Las semillas de Randia armata (Sw.) DC. tuvieron el embrión más pequeño, mientras que C. platanifolia presentó uno de los embriones más grandes, alcanzando un tamaño de $38.2 \mathrm{~mm}$ de largo.

Rasgos cualitativos: Siete de los 11 rasgos cualitativos medidos mostraron diferencias significativas en sus frecuencias (Cuadro 3). La mayoría de las especies ( $66 \%)$ de bosque

\section{CUADRO 3}

Análisis Chi cuadrado de frecuencias en rasgos morfológicos cualitativos

TABLE 3

Chi-square analysis of frequencies within qualitative morphological traits

\begin{tabular}{lccc}
\multicolumn{1}{c}{ Rasgo cualitativo } & $\mathrm{X}^{2}$ & $\mathrm{gl}$ & $\mathrm{p}$ valor \\
Apéndice & 7.91 & 1 & 0.005 \\
Areola & 32.92 & 1 & 0.001 \\
Forma & 148.34 & 7 & 0.001 \\
Endospermo & 8.12 & 1 & 0.004 \\
Tipo de embrión & 41.99 & 5 & 0.001 \\
Tipo dispersión & 7.92 & 2 & 0.019 \\
Tipo de fruto & 13.79 & 1 & 0.001 \\
\hline
\end{tabular}

gl = grados de libertad 

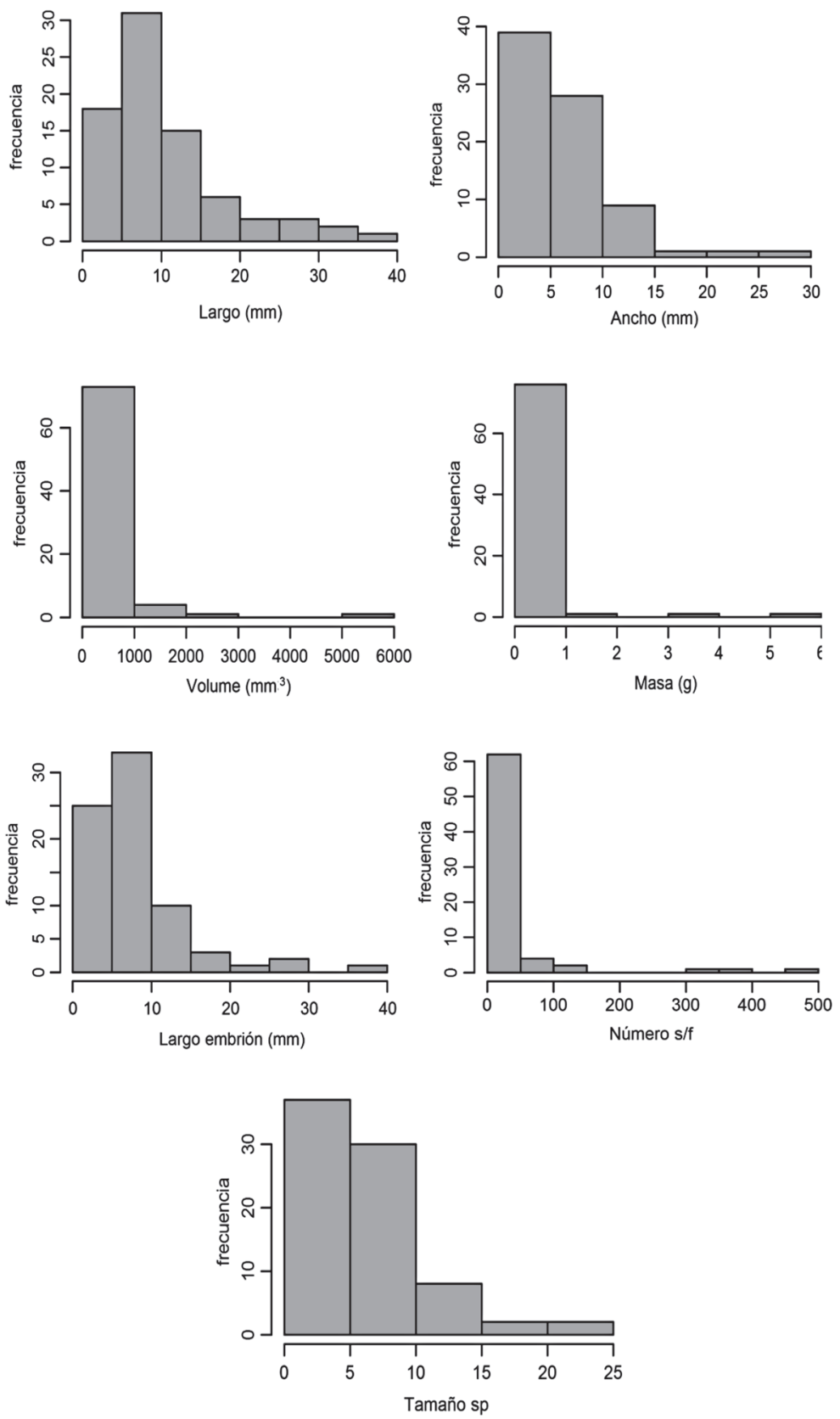

Fig. 1. Distribución de frecuencias de rasgos cuantitativos en las semillas de una comunidad de especies leñosas de bosque seco tumbesino.

Fig. 1. Frequency distribution of quantitative traits in seeds of a dry forest community from the Tumbesian region. 


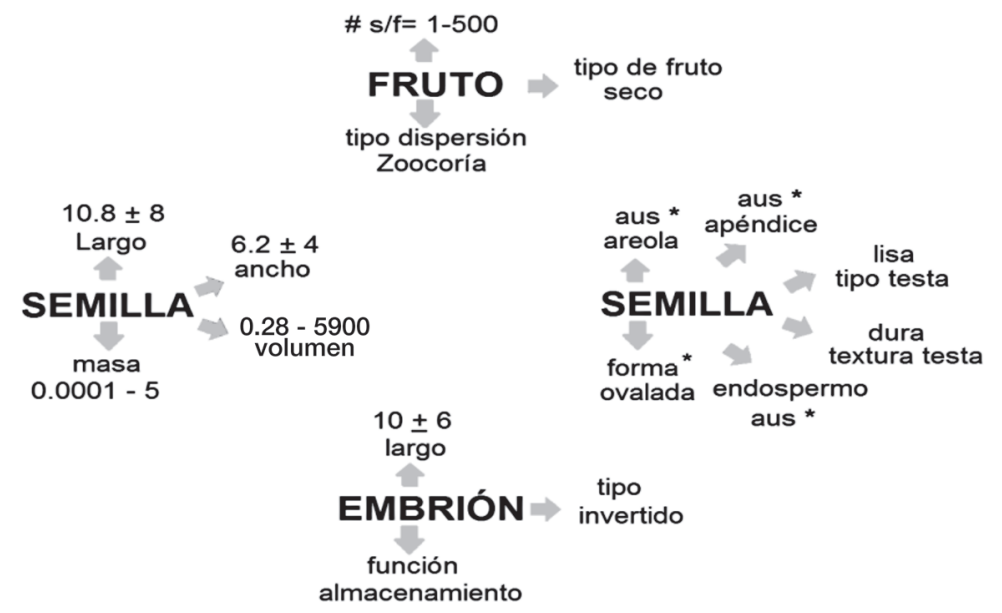

Fig. 2. Rasgos morfológicos característicos en una comunidad de especies leñosas de bosque seco. Significado de \# s/f= número de semillas por fruto; aus $=$ ausente. El asterisco $(*)$ representa estadísticamente significativo $(\mathrm{p}<0.05)$.

Fig. 2. Morphological traits characteristic of a community of woody species from dry forest. Significancy of \# S / f = number of seeds per fruit; aus $=$ absent. The asterisk $(*)$ represents statistically significant $(\mathrm{p}<0.05)$.

secos no presentaron algún tipo de apéndices o areola (80\%) en sus semillas (Fig. 3). La mayoría de las especies se caracterizaron por tener semillas ovaladas y sin endospermo, la reserva nutritiva se encuentra en los cotiledones de los embriones; sin embargo, al analizar la función de embriones, ya sea como de almacenamiento o fotosintéticos (foliares) no se encontró diferencias significativas entre estos dos rasgos. De igual manera con respecto a la textura y tipo de testa de las semillas, no existieron diferencias significativas entre sus frecuencias, pero la tendencia de las especies es producir semillas con testa dura y lisa. En las semillas encontramos solamente séis tipos de embriones (doblado, espatulado, invertido, linear, periférico y plegado) de los 12 clasificados por Martin (1946), el $40 \%$ de las especies posee semillas con embriones invertidos (ej. Caesalpinia spinosa, Geofroea spinosa, Hura crepitans, Cercidium praecox, Pithecelobium excelsum, Ziziphus thyrsiflora entre otras especies), seguido del $25 \%$ de las especies con embriones espatulados en la que se destacan especies como; Carica parviflora, Cedrela odorata, Lafoencia acuminata, Erythoyxylum galucum, Ochroma pyramidale entre otras. La dispersión de semillas está dominada por zoocoria (Fig. 3) en un $38 \%$ con relación a anemocoria (22\%) y autocoria (19\%). Sin embargo, encontramos que el $70 \%$ de las especies posee frutos secos como por ejemplo en Coccoloba ruiziana, Cochlospermum vitifolium, Erythrina velutina, Gallesia integrifolia. En cambio especies como Acnistus arborescens, Bursera graveolens, Carica parviflora, Celtis iguanaea, Geogroea spinosa, Psidium guayava tuvieron frutos carnosos.

Diferencia de masa con otros bosques: La distribución de los valores de la masa en las semillas de los diferentes bosques mostró gran variabilidad, encontrando especies con semillas desde $0.1 \mathrm{~g}$ a más de $35 \mathrm{~g}$. Al comparar la masa de las semillas de bosque seco con respecto a los otros tipos de bosques tropicales, los análisis mostraron que estas especies poseen menor peso que las especies de los otros bosques (Fig. 4), el BHT presentó especies con las semillas de mayor peso (Fig. 5), llegando a tener especies con semillas hasta de $35 \mathrm{~g}$. Las diferencias significativas (Kruskal-Wallis, $\mathrm{p}<0.05$ ) de la masa entre las especies de los diferentes bosques se muestran en el cuadro 4. 

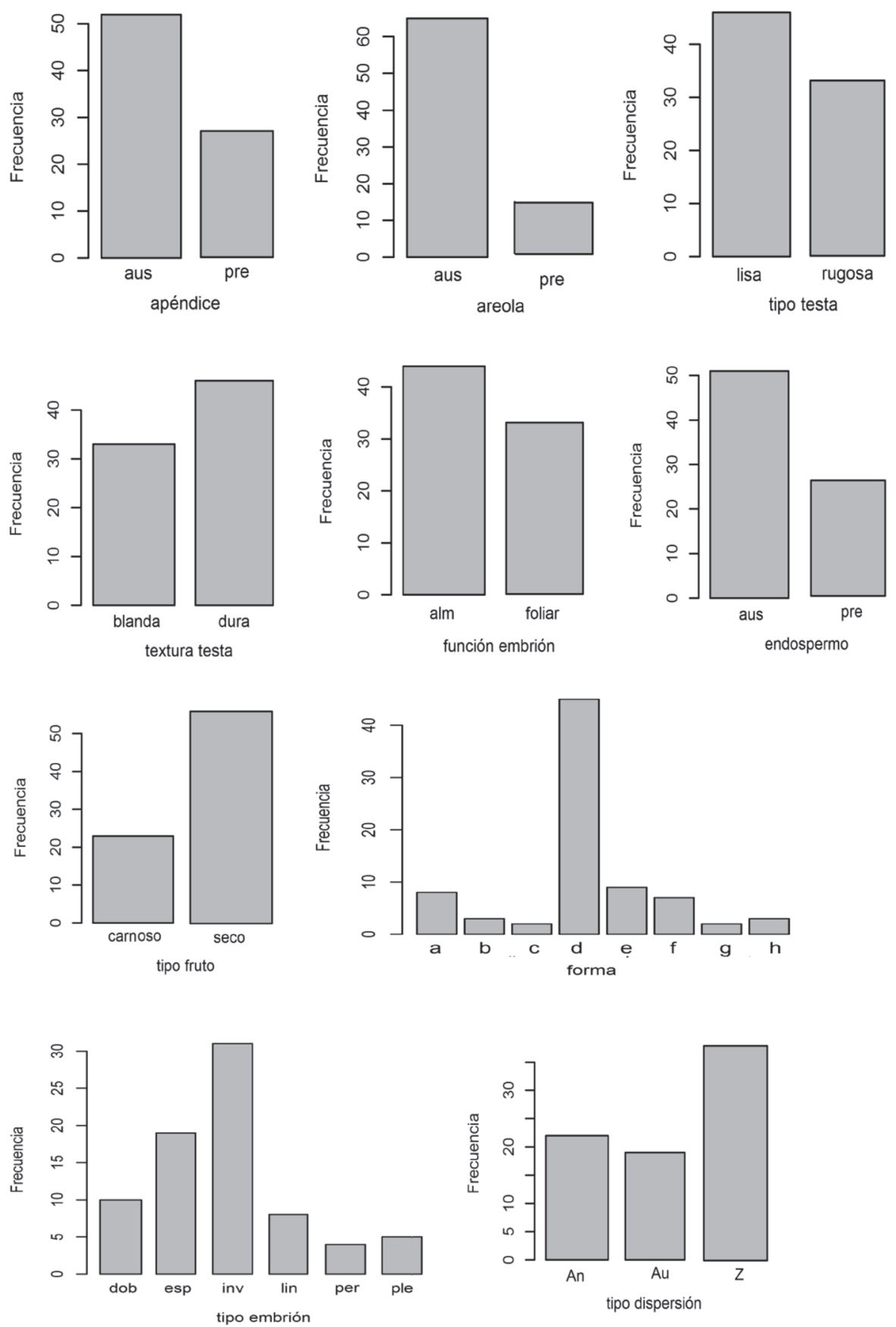

Fig. 3. Distribución de Frecuencias de rasgos cualitativos en semillas de especies de bosque seco. Formas de semillas: a = alada; $\mathrm{b}=$ elíptica; $\mathrm{c}=$ linear; $\mathrm{d}=$ ovalada; $\mathrm{e}=$ redonda; $\mathrm{f}=$ reniforme; $\mathrm{h}=$ triangular. Tipo de embrión: dob $=$ doblado; esp $=$ espatulado; inv $=$ invertido; per $=$ periférico; ple $=$ plegado. Tipo de dispersión: $A n=$ anemocoria; $A u=A u t o c o r i a ; Z=$ zoocoria; aus = ausente; pre $=$ presente.

Fig. 3. Frequency distribution of qualitative traits in seeds of dry forest species. Seed forms: $a=$ winged; $b=$ elliptical; $c$ $=$ linear; $\mathrm{d}=$ oval; $\mathrm{e}=$ round; $\mathrm{f}=$ reniforme; $\mathrm{h}=$ triangular. Embryo type: $\mathrm{dob}=$ bent; esp = spatulate; inv = investing; per $=$ peripheral; $\mathrm{ple}=$ folded. Dispersion type: $\mathrm{An}=$ anemochory; $\mathrm{Au}=$ autochory; $\mathrm{Z}=$ zoochory; aus $=$ ausent; pre $=$ present. 

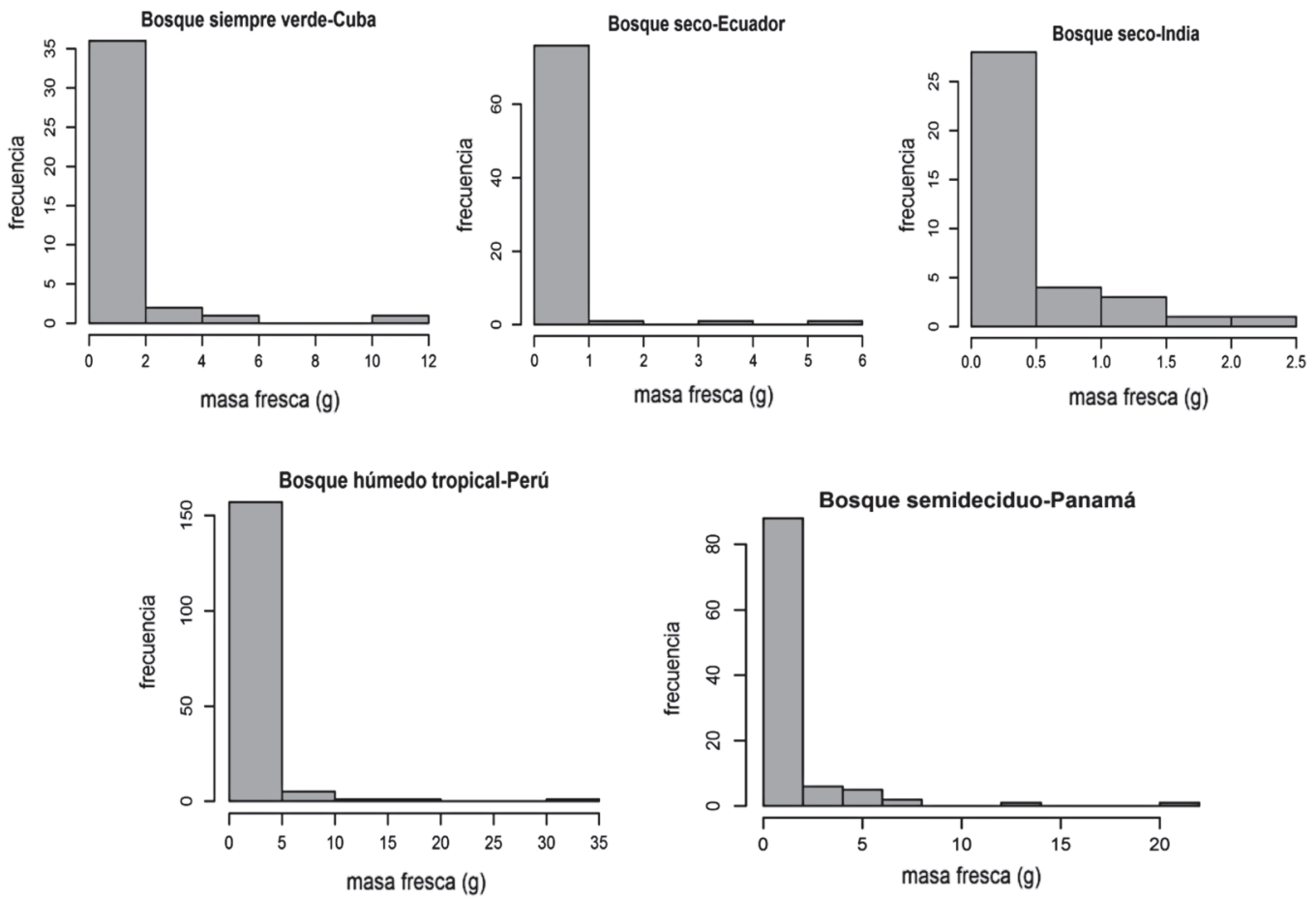

Fig. 4. Distribución de frecuencias de la masa de las semillas en cinco bosques tropicales.

Fig. 4. Frecuency distribution of seed mass in five tropical forests.

\section{CUADRO 4}

Análisis de comparación multiple entre la masa de semillas en diferentes tipos de bosques

TABLE 4

Multiple comparisons tests of seed mass from different types of forests

\begin{tabular}{lccc}
\multicolumn{1}{c}{ Tipos de bosque } & Valor observado & Valor crítico & Diferencia \\
BHT-BSD & 49.25 & 43.19 & sig \\
BHT-BS* & 91.72 & 47.06 & sig \\
BHT-BS & 77.09 & 62.57 & sig \\
BHT- BSV & 61.87 & 60.62 & sig \\
BSD-BS & 42.47 & 51.44 & $\mathrm{~ns}$ \\
BSD-BS & 27.84 & 65.92 & $\mathrm{~ns}$ \\
BSD-BS & 12.61 & 64.92 & $\mathrm{~ns}$ \\
BS-BS & 14.62 & 68.52 & $\mathrm{~ns}$ \\
BS-BSV & 29.85 & 66.75 & $\mathrm{~ns}$ \\
BS-BSV & 15.22 & 78.46 & $\mathrm{~ns}$ \\
\hline
\end{tabular}

Significado de BHT = Bosque Húmedo tropical; BSD = Bosque Semi Deciduo; BS = Bosque seco; BSV = Bosque siempre verde. sig representa diferencias significativas $(p<0.05)$ y ns no significativo, el asterico $(*)$ representa bosque seco en estudio.

Significancy of BHTmf = Tropical Rainforest BSD $=$ SemiDeciduous Forest; BS $=$ Dry forest; BSV $=$ Evergreen forest. 


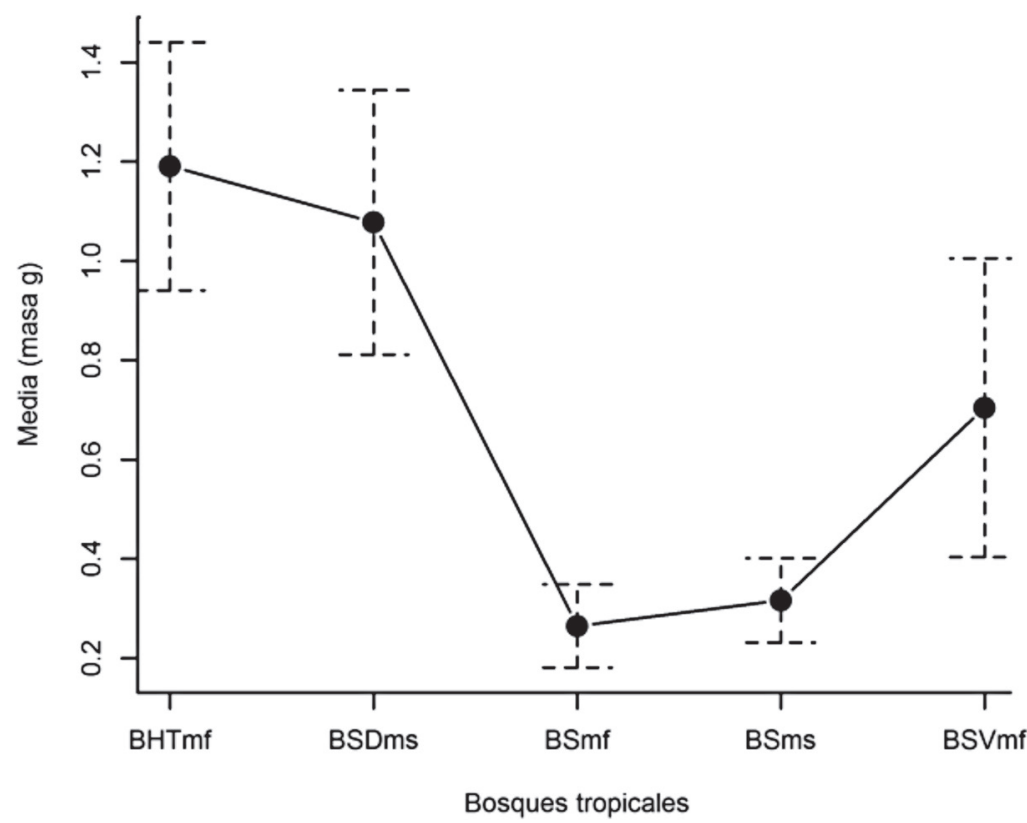

Fig. 5. Diferencia de medias ( \pm error típico) de la masa de las semillas entre bosques: BHTmf = Bosque Húmedo tropical masa fresca, $\mathrm{BSDms}=$ Bosque Semi Deciduo masa seca, BSms= Bosque seco masa seca, BSVmf = Bosque siempre verde masa fresca.

Fig. 5. Mean Difference ( \pm standard error) of seed mass between forest: BHTmf $=$ Tropical Rainforest, fresh mass; BSDms $=$ SemiDeciduous Forest, dry mass; BSms $=$ Dry forest, dry mass; BSVmf $=$ Evergreen forest, fresh mass.

\section{DISCUSIÓN}

Los estudios de rasgos funcionales de los bosques secos neotropicales se han limitado a conocer la diversidad de especies, estatura, complejidad, productividad primaria neta entre otros rasgos (Sánchez-Azofeifa, Powers, Fernandes, \& Quezada, 2014), pero pocos estudios se han realizado sobre rasgos regenerativos para toda una comunidad de especies leñosas. Nuestros resultados mostraron que existe gran heterogeneidad en la mayoría de los rasgos, lo que podría determinar una variedad de comportamientos y características en semillas de las especies de bosque seco. La variación en el tamaño, masa y volumen de las semillas de las especies en estudio, puede indicar que estas especies manifiesten una buena permanencia en el suelo y al hábitat donde se desarrollan (Yu, Sternberg, Kutiel, \& Chen, 2007), además de una amplia respuesta adaptativa a las condiciones ambientales de ambientes áridos. Sin embargo, encontramos que la tendencia de la mayoría de especies es producir semillas más pequeñas con respecto a la masa encontrada en otros bosques del trópico, contrario a la percepción general que se tiene que las especies de zonas tropicales poseen semillas grandes, por lo tanto, basado en nuestros resultados, la evidencia de una asociación entre semillas grandes y hábitats secos sigue siendo limitada (Leishman, Wright, Moles, \& Westoby, 2000). El tamaño pequeño de las semillas encontrado en las especies, puede otorgar ciertas ventajas ecológicas en los bosques secos, ya que las semillas pequeñas tienen mejor oportunidad para penetrar en el suelo fácilmente y formar bancos de semillas (Narayan \& Pandey, 2014), permitiéndoles sobrevivir a una mayor cantidad de micrositios, crucial para la regeneración de las especies luego de una perturbación (Khurana \& Singh, 2001). Además, un tamaño pequeño reduce la superficie de contacto con 
factores ambientales, lo que podría significar que las semillas de las especies de bosque seco al ser pequeñas, podrían reducir su muerte por deshidratación en ambientes áridos.

Si bien, las especies de bosque seco mostraron un amplio rango en el número de semillas por fruto, la tendencia de la mayoría de especies es producir entre uno a diez semillas/ fruto. En ambientes secos, las plantas podrían destinar mayor cantidad de energía a la producción de una sola o pocas semillas para que pudiesen tener mayor probabiliad de tolerar las condiciones de aridez de estos ambientes. En cambio, la producción elevada de semillas en algunas especies, se justifica como una estrategia reproductiva, debido a que si existe una mayor cantidad de semillas, hay mayor probabilidad de que alguna se establezca en sitios favorables para su germinación y crecimiento, cuando las condiciones climáticas sean propicias para ello (Ayala-Cordero, Terraza, Lépez-Mata, \& Trejo. 2004).

Nuestros resultados mostraron que las especies leñosas de bosque seco presentan rasgos morfológicos cualitativos dominates en las semillas, encontramos que la mayoría de especies presentan dispersión por animales, resultados similares a los encontrado por $\mathrm{Du}$, Mi, Liu, Chen y Ma (2009) y Jara-Gerrero et al. (2011) en especies tropicales. Encontrar más especies dispersadas por zoocoria se convertiría en un patrón para los bosques secos neotropicales y tumbesinos. No obstante, en especies presentes en una zona semiárida del Noreste de Brazil, solo encontraron el $22 \%$ de las especies dispersada por animales (Silva, Lopes, \& Miranda de Melo, 2015). En zonas áridas, la zoocoria podría ayudar a las semillas a tener más opciones para alejarse de la planta madre, evitar la alta mortalidad (Griz \& Machado, 2001) y buscar nuevos ambientes para sobrevivir y germinar. Sin embargo, en contraste con el tipo de dipersión, nuestro estudio determinó que la mayor cantidad de especies posee frutos secos, cuando la norma dicta que la dispersión por animales se da en los frutos carnosos (Seidler \& Plotkin, 2006). La escasez de frutos carnosos durante la estación seca en este tipo de vegetación podría forzar una amplia gama de animales a alimentarse de cualquier tipo de fruta que está disponible (Griz \& Machado, 2001), razón por la cual encontramos la mayor cantidad de especies zoocoras.

La mayoría de especies también se caracterizaron por no presentar algún tipo de apéndice, areola o endospermo, siendo los cotiledones quienes cumplen la función de almacenamiento de reservas, estos rasgos pudiesen tener grandes connotaciones ecológicas en el bosque seco, un embrión desarrollado y sin endospermo en la mayoría de especies podría ayudar a acelerar la germinación en los cortos periodos de lluvias que tienen estos bosques. El endospermo no se constituiría en barrera para el crecimiento del embrión como sucede en otras especies (Baskin \& Baskin, 2005; Yan, Duermeyer, Leoveanu, \& Nambara, 2014). La relación entre el embrión y la germinación ya ha sido probado en otros estudios, existiendo un aumento de la tasa de germinación y disminución de la latencia al aumentar la longitud del embrión (Baskin \& Baskin, 2004; Vandelook et al., 2012b) a diferencia de las especies con una menor longitud del embrión, que poseen una latencia superior y necesitan más tiempo para desarrollarse y germinar (Vandelook et al., 2012b). Como sabemos, el desarrollo embrionario varía considerablemente entre las especies y con frecuencia ofrece caracteres taxonómicos valiosos, lo que se traduce en una amplia variación en el tamaño, forma y disposición del embrión en la madurez de la semilla (Dickie \& Stuppy, 2003). Nosotros identificamos que la mayor cantidad de especies en bosque seco, poseen embriones grandes, gruesos e invertidos que ocupan casi todo el interior de las semillas, considerados como más avanzados y desarrollados evolutivamente con respecto a otros tipos de embriones (Forbis, Floyd, \& De Queiroz, 2002; Vandelook et al., 2012b); en cambio, embriones subdesarrolados podrían generar algún tipo de dormancia y retrasar la germinación (Baskin \& Baskin, 2005; Finch-Savage \& LeubnerMetzger, 2006); sin embargo, en especies de bosque seco, los diferentes tipos de dormancia posiblemente son una estrategia favorable que 
les permite a las especies sobrevivir como semillas, hasta cuando las condiciones ambientales sean favorables para germinar.

En este estudio, se encontró que la producción de semillas lisas y rugosas en las especies de bosque seco se da en igual proporción, resultados similares se dieron entre semillas de testa dura y blanda, pero la tendencia ligera de la mayoría de especies es tener semillas con testa lisa y dura. Estas características, junto con el tamaño de las semillas sería fundamental para protegerse de la deshidratación generada por las altas temperaturas en los bosques secos; una testa dura impediría la pérdidad de agua en las semillas. Sin embargo, la presencia de testa dura también puede provocar dormancia física en semillas de algunas especies (Finch-Savage \& Leubner-Metzger, 2006). El tipo de dormacia ocacionada por la cubierta de la semilla en zonas áridas es parte de una de las estrategia de supervivencia de semillas de muchas especies (Baskin \& Baskin, 2014), al actuar como un controlador de la germinación (Finch-Savage \& Leubner-Metzger, 2006). Esto sería importante en las semillas, al momento de enfrentar condiciones ambientales adversar presentes en los bosques secos y germinar solo cuando se crean las condiciones ambientales necesarias para hacerlo, por lo tanto, la producción de semilla dura es un mecanismo de supervivencia y un rasgo para explicar la persistencia de semillas en el suelo (Gardarin, Durr, Mannino, Busset, \& Colbach, 2010). Con respecto a la forma de las semillas, la tendencia de las especies de bosque seco es producir semillas ovaladas y redondas, esto podría influir en las posibilidades de colonización de un determinado micro sitio (Khurana \& Singh, 2001; Fenner \& Thompson, 2005) y en la longevidad que pueden alcanzar la semillas en el suelo, luego de haber sido dispersadas (Khurana \& Singh, 2001); al parecer las semillas más redondas sobreviven más tiempo que las angulares (Dalling, 2002).No obstante, esto difiere con otros estudios, donde la forma de la semilla parece no tener tan buen valor predictivo en relación con la persistencia y su efecto sobre el entierro es presumiblemente dependiente sobre todo del tamaño (Peco, Traba, Levassor, Sánchez, \& Azcarate, 2003). Una desventaja de las especies del bosques seco al tener semillas ovaladas o redondas, es que pueden correr el riesgo de influir de manera indirecta a la infestación por algunos insectos, como ocurren con ciertas especies de Fabaceae (Johnson \& Romero, 2004). En conclusión, nuestros resultados mostraron que existe gran variabilidad en los rasgos morfológicos regenerativos de las especies leñosas del bosque seco, lo que les permitirá tener un amplio rango de mecanismos para soportar condiciones de estrés ambiental en las zonas áridas. Además, encontramos que existen determinados rasgos morfológicos en las semillas con grandes implicaciones ecológicas, que les significaría a las especies estar mejor preparadas para soportar los cambios ambientales globales pronosticados para los próximos años. Finalmente, nuestros resultados aportan con información fundamental para mejorar nuestra compresión del rol ecológico de las semillas en la dinámica de los bosques secos y genera indicadores morfológicos para la gestión y conservación ex situ de las especies leñosas de zonas secas tropicales.

\section{AGRADECIMIENTOS}

Este trabajo contó con el apoyo financiero del programa de becas SENESCYT-Ecuador 2008-2 y por proyectos internos de la Universidad Técnia Particular de Loja. Gracias por el apoyo al personal que ha formado parte del Banco de Germoplasma de la UTPL durante la recolección de semillas.

\section{RESUMEN}

El estudio de rasgos morfológicos funcionales nos permite conocer muchos aspectos fundamentales de la dinámica de las comunidades vegetales en hábitats particulares y a nivel mundial, los rasgos morfológicos regenerativos cumplen un rol importante en la ecología e historia de las plantas por estar relacionados con la dispersión, germinación, colonización y establecimiento de las plántulas en determinados hábitas, sin embargo estos rasgos no han sido debidamente estudiados a nivel de toda una comunidad de especies leñosas en los bosques secos 
neotropicales. Los objetivos del presente estudio fueron; a) evaluar rasgos morfológicos funcionales en frutos, semillas y embriones de especies leñosas, b).- determinar que patrones morfológicos caracterizan a las semillas de la comunidad de especies leñosas y c) analizar la masa de la semillas con respecto a otros bosques tropicales. Entre el 2010 y 2014 se recolectaron en un bosque seco tumbesino ubicado al sur occidente del Ecuador frutos con semillas maduras de 79 especies pertenecientes a 42 árboles y 37 arbustos de 31 familias más representativas de los bosques secos tumbesinos. Se midió y describió un total de 18 rasgos morfológicos, 7 cuantitativos y 11 cualitativos en frutos, semillas y embriones. La descripción y análisis de los rasgos se realizaron en el Banco de Germoplasma de la UTPL. Los resultados mostraron gran heterogeneidad en los rasgos cuantitativos medidos, el tamaño de las semillas varió de 1.3 a $39 \mathrm{~mm}$ de largo x 0.6 a $25 \mathrm{~mm}$ de ancho, mientras que el promedio del largo de los embriones fue de $8.1 \mathrm{~mm}$. El volumen, masa y número de semillas por fruto fueron los rasgos que presentaron mayor variabilidad. $\mathrm{La}$ mayoría de especies de bosque seco se caracterizaron por presentar frutos secos dispersados por animales, con semillas ovaladas sin areola, lisas y de testa dura. Encontramos solamente seis tipos de embriones en las semillas, el 40 $\%$ de las especies se caracterizó por presentar embriones invertidos bien desarrollados con cotiledones grandes y gruesos que ocupan todo el interior de la semillas y cumplen la función de almacenamiento de reservas nutritivas. En conclusión, existe gran variabilidad y heterogeneidad en los rasgos morfológicos de las semillas de especies leñosas del bosque seco, lo que les permitiría tener un amplio rango de mecanismos y comportamiento para soportar condiciones de estrés ambiental en zonas áridas. Las implicaciones ecológicas que podrían tener los rasgos que caraterizan a los frutos, semillas y embriones de la comunidad de especies leñosas posiblemente les permitan estar mejor preparadas para soportar los cambios climáticos globales pronosticados para los próximos años.

Palabras clave: bosque seco tropical, árboles, arbustos, embriones vegetales, frutos, Ecuador

\section{REFERENCIAS}

Aide, T. M., Clark, M. L., Grau, H. R., López-Carr, D., Levy, M. A., Redo, D., ...Muñiz, M. (2012). Deforestation and reforestation of Latin America and the Caribbean (2001-2010). Biotropica, 45(2), 262-71.

Andrew, N. R., Hart, R. A., Jung, M. P., Hemmings, Z., \& Terblanche, J. S. (2013). Can temperate insects take the heat? A case study of the physiological and behavioural responses in a common ant, Iridomyrmex purpureus (Formicidae), with potential climate change. Journal of Insect Physiology, 59, 870-880.
Aguirre, Z. \& Kvist, L. P. (2005). Composición florística y estado de conservación de los bosques secos del suroccidente del Ecuador. Lyonia, 8, 41-67.

Aguirre, Z., Linares-Palomino, R., \& Kvist, L. (2006). Especies leñosas y formaciones vegetales en los bosques estacionalmente secos de Ecuador y Perú. Arnaldoa, 13, 324-350.

Ayala-Cordero, G., Terrazas, T., López-Mata, L., \&Trejo, C. (2004). Variación en el tamaño y peso de la semilla y su relación con la germinación en una población de Stenocereus beneckei. Interciencia, 29, 692-697.

Baskin, J. M., \& Baskin, C. C. (2004). A classification system for seed dormancy. Seed Science Research, 14, 1-16.

Baskin, C. C., \& Baskin, J. M. (2005). Underdeveloped embryos in dwarf seeds and implications for assignment to dormancy class. Seed Science Research, $15,357-360$.

Baskin, C. C., \& Baskin, J. M. (2014). Seeds: Ecology, biogeography and evolution of Dormancy and Germination ( $2^{\text {nd }}$ Ed.). Kentucky, USA: Elsevier.

Bihn, J. H., Gebauer, G., \& Brandl, R. (2010). Loss of functional diversity of ant assemblages in secondary tropical forests. Ecology, 91, 782-792.

Blackie, R., Baldauf, C., Gautier, D., Gumbo, D., Kassa, H., Parthasarathy, N.,..Sunderland, T. (2014). Tropical dry forests: The state of global knowledge and recommendations for future research (Discussion Paper). Bogor, Indonesia: CIFOR.

Chaturvedi, R. K., Raghubanshi, A. S., \& Singh, J. S. (2011). Leaf attributes and tree growth in a tropical dry forest. Journal of Vegetation Science, 22(5), 917-931.

Dalling, J. W. (2002). Ecología de semillas. In M. Guariguata \& G. Kattan (Eds.), Ecología y conservación de bosques neotropicales (pp. 346-375). Costa Rica: Ediciones LUR.

Daws, M. I., Garwood, N. C., \& Pritchard, H. W. (2006). Prediction of desiccation sensitivity in seeds of woody species: a probabilistic model based on two seed traits and 104 species. Annals of Botany, 97, 667-74.

Dickie, J. B., \& Stuppy, W. H. (2003). Seed and Fruit Structure: significance in seed conservation operations. In R. D. Smith, J. B. Dickie, S. H. Linington, H. W. Pritchard, \& R. J. Probert (Eds.), Seed conservation: turning science into practice. London: The Royal Botanic Gardens, Kew.

Donohue, K., Rubio de Casas, R., Burghardt, L., Kovach, K., \& Willis, C. G. (2010). Germination, postgermination adaptation, and species ecological ranges. 
Annual Review of Ecology, Evolution, and Systematics, 41, 293-319.

Du, Y., Mi, X., Liu, X., Chen, L., \& Ma, K. (2009). Seed dispersal phenology and dispersal syndromes in a subtropical broad-leaved forest of China. Forest Ecology and Management, 258, 1147-1152.

Espinosa, C. I., Cabrera, O., Luzuriaga, A., \& Escudero, A. (2011). What Factors Affect Diversity and Species Composition of Endangered Tumbesian Dry Forests in Southern Ecuador? Biotropica, 43, 15-22.

Espinosa, C., De la Cruz, M., Luzuriaga, A., \& Escudero, A. (2012). Escudero Bosques tropicales secos de la región Pacífico Ecuatorial: diversidad, estructura, funcionamiento e implicaciones para la conservación. Ecosistemas, 21, 167-179.

Fenner, M., \& Thompson, K. (2005). The ecology of seeds. Cambridge, UK: Cambridge University Press.

Finch-Savage, W. E., \& Leubner-Metzger, G. (2006). Seed dormancy and the control of germination. New Phytologist, 171, 501-523.

Forbis, T. A., Floyd, S. A., \& De Queiroz, A. (2002). The evolution of embryo size in angiosperms and other seed plants: implications for the evolution of seed dormancy. Evolution, 56, 2112-2125.

Foster, S., \& Janson, C. H. (1985). The relationship between seed size and establishment conditions in tropical woody plants. Ecology, 66, 773-780.

Gardarin, A., Durr, C., Mannino, M. R., Busset, H., \& Colbach, N. (2010). Seed mortality in the soil is related to seed coat thickness. Seed Science Research, 20, 243-256.

Garnier, E., Cortez, J., Billés, G., Navas, M. L., Roumet, C., Debussche, M.,... Toussaint, J. P. (2004). Plant functional markers capture ecosystem properties during secondary succession. Ecology, 85(9), 2630-2637.

Griz, L. M., \& Machado, I. C. (2001). Fruiting phenology and seed dispersal syndromes in Caatinga, a tropical dry forest in the northeast of Brazil. Journal of Tropical Ecology, 17, 303-321.

Gritti, E. S., Cassignat, C., Flores, O., Bonnefille, R., Chalié, F., Guiot, J., \& Jolly, D. (2010). Simulated effects of a seasonal precipitation change on thevegetation in tropical Africa. Climate of the Past, 6, 169-178.

Jara-Guerrero, A., De la Cruz, M., \& Méndez, M. (2011). Seed Dispersal Spectrum of Woody Species in South Ecuadorian Dry Forests: Environmental Correlates and the Effect of Considering Species Abundance. Biotropica, 43, 722-730.
Johnson, C., \& Romero, J. (2004). A review of evolution of oviposition guilds in the Bruchidae (Coleoptera). Revista Brasileira de Entomologia, 48(3), 401-408.

Kitajima, K. (1996). Cotyledon functional morphology, patterns of seed reserve utilization and regeneration niches of tropical tree seedlings. In M. D. Swaine (Ed.), The ecology of tropical forest tree seedlings (pp. 193-210). Paris, France: Parthenon Publishing Group.

Khurana, E., \& Singh, J. S. (2001). Ecology of seed and seedling growth for conservation and restoration of tropical dry forest: a review. Environmental Conservation, 28(1), 39-52.

Khurana, E., Sagar, R., \& Singh, J. S. (2006). Seed size: a key trait determining species distribution and diversity of dry tropical forest in northern India. Acta Oecologica, 29, 196-204.

Kröber, W., Böhnke, M., Welk, E., Wirth, C., \& Bruelheide, H. (2012). Leaf trait-environment relationships in a subtropical broadleaved forest in South-East China. PLOS ONE, 7(4), 1-11.

Laughlin, D. C., Joshi, C., Van, P. M., Bastow, Z. A., \& Fulé, P. Z. (2012). A predictive model of community assembly that incorporates intraspecific trait variation. Ecology Letter, 15(11), 1291-1299.

Leishman, M. R., Wright, I. J., Moles, A. T., \& Westoby, M. (2000). The Evolutionary Ecology of Seed Size. In M. Fenner, (Ed.), Seeds: the ecology of regeneration in plant communities (pp. 31-57). Wallingford, UK: CABI Publishing.

Linares-Palomino, R., Oliveira-Filho, A. T., \& Pennington, R. T. (2011). Neotropical seasonally dry forests: diversity, endemism and biogeography of woody plants. In R. Dirzo, H. Mooney, G. Ceballos \& H. Young (Eds.), Seasonally Dry Tropical Forests: Biology and conservation (pp. 3-21). Washington D.C.: Island Press.

Linares-Palomino, R., Kvist, L. P., Aguirre-Mendoza, Z., $\&$ Gonzales-Inca, C. (2010). Diversity and endemism of woody plant species in the Equatorial Pacific seasonally dry forests. Biodiversity and Conservation, 19, 169-185.

Narayan, D., \& Pandey, N. (2014). Tropical Dry Forest Restoration: Science and Practice of Direct Seeding in a Nutshell (Occaional paper). Rajasthan State Pollution Control Board, 7, 2-19.

Newton, T. Marchese, J. A., Fernandes de Sousa, A. K., Curti, G. L., Fogolari, H., \& Vinicius, S. (2013). Uso do software ImageJ na estimativa de área foliar para a cultura do feijão. Interciencia, 38, 843-848.

Martin, A. C. (1946). The comparative internal morphology of seeds. American Midland Naturalist, 36, 513-660. 
Metz, J., Liancourt, P., Kigel, J., Harel, D., Sternberg, M., \& Tielborger, K. (2010). Plant survival in relation to seed size along environmental gradients: a long-term study from semi-arid and Mediterranean annual plant communities. Journal of Ecology, 98, 697-704.

Peco, B., Traba, J., Levassor, C., Sánchez, A. M., \& Azcarate, F. M. (2003). Seed size, shape and persistence in dry Mediterranean grass and scrublands. Seed Science Research, 13(1), 87-95.

Pennington, T., Lewis, G., \& Ratter, J. (2006). Neotropical savannas and seasonally dry forests: plant diversity, biogeography and conservation. Florida: CRC Press

Portillo-Quintero, C., \& Sánchez-Azofeifa, G. (2010). Extent and conservation of tropical dry forests in the Americas. Biological Conservation, 143(1),144-155.

Quesada, M., \& Stoner, K. E. (2004). Threats to the conservation of tropical dry forest in Costa Rica. In G. W. Frankie, A. Mata \& V. S. Brandleigh (Eds.), Biodiversity Conservation in Costa Rica: Learning the Lessons in a Seasonal Dry Forest (pp. 266-280). Berkeley: University of California Press.

R Core Team. (2013). R: A language and environment for statistical computing. Vienna, Austria: R Foundation for Statistical Computing. Retrieved from http:// www.R-project.org/

Sánchez-Azofeifa, A., Powers, J., Fernandes, G., \& Quezada, M. (Eds.). (2014). Tropical Dry Forests in the Americas. Ecology, Conservation, and Management. New York: CRC Press.

Sánchez, J .A., Muñoz, B., \& Montejo, L. (2009). Rasgos de semillas de árboles en un bosque siempreverde tropical de la Sierra del Rosario, Cuba. Pastos y Forrajes, 32, 1-20.

Seidler, T. G., \& Plotkin, J. B. (2006). Seed dispersal and spatial pattern in tropical trees. PLoS Biology, 4(11), 2132-2137.
Silva, E. C., Lopes, S. D., \& Miranda de Melo, J. I. (2015). Floristic similarity and dispersal syndromes in a rocky outcrop in semi-arid Northeastern Brazil. Revista de Biología Tropical, 63(3), 827-843.

Scholze, M., Knorr, W., Arnell, N. W., \& Prentice, I. C. (2006). A climate-change risk analysis for world ecosystems. The National Academy of Sciences of the USA, 103(35), 13116-13120.

Shipley, B., Vile, D., \& Garnier, E. (2006). From plant traits to plant communities: A statistical mechanistic approach to biodiversity. Science, 314, 812-814.

The IUCN. (2015). Red List of Threatened Species. Version 2015.2. Retrieved from www.iucnredlist.org.

Vandelook, F., Verdu, M., \& Honnay, O., (2012a). The role of seed traits in determining the phylogenetic structure of temperate plant communities. Annals of Botany, 110(3), 629-636.

Vandelook, F., Janssens, S. B., \& Probert, R. J., (2012b) Relative embryo length as an adaptation to habitat and life cycle in Apiaceae. New Phytologist, 195(2), 479-487.

Westoby, M., Falster, D. S., Moles, A. T., Vesk, P. A., \& Wright, I. J. (2002). Plant ecological strategies: some leading dimensions of variation between species. Annual Review of Ecology, Evolution and Systematics, 33, 125-59.

Yan, D., Duermeyer, L., Leoveanu, C., \& Nambara, E. (2014). The Functions of the Endosperm During Seed Germination. Plant \& Cell Physiology, 9, 1521-33.

Yates, M. L., Andrew, N. R., Binns, M., \& Gibb, H. (2014). Morphological traits: predictable responses to macrohabitats across a $300 \mathrm{~km}$ scale. PeerJ, 2, e271.

Yu, S., Sternberg, M., Kutiel, P., \& Chen, H. (2007). Seed mass, shape, and persistence in the soil seed bank of Israeli coastal sand dune flora. Evolutionary Ecology Research, 9(2), 325-340. 
